\title{
NOUVELLE
}

\section{Arginine et réponse immunitaire innée}

\section{Au-delà de la production de monoxyde d'azote}

Virginie Mieulet, Richard F. Lamb

> La qualité de la réponse immunitaire innée engendrée par des agents pathogènes est étroitement liée au statut nutritionnel d'un individu. Des études épidémiologiques ont révélé une corrélation entre malnutrition et susceptibilité d'un individu aux infections [1]. De plus, il apparaît clairement que la survie des animaux infectés par des microorganismes est dépendante de leur état nutritionnel [2, 3]. Par exemple, un régime pauvre en protéines chez des souris atteintes de tuberculose entraîne une réaction inflammatoire inadaptée et compromet leur survie [3]. Cependant, les mécanismes impliqués dans cette relation de cause à effet entre nutrition et infection ne sont pas clairs et aucun nutriment particulier ayant un rôle prépondérant n'a été identifié.

La détection de pathogènes par la voie de signalisation des récepteurs Toll-like (TLR) est un élément majeur de la réponse immunitaire innée car elle induit l'activation des macrophages [4]. En effet, le
LPS (lipopolysaccharide), un composant de la paroi des bactéries Gram négatives, interagit avec le TLR4, ce qui conduit à l'activation des cascades de signalisation MAPK (mitogen activated protein kinase), NFKB (nuclear factor $\kappa B$ ), JNK (c-Jun N-terminal kinase) et p38MAPK dans les macrophages. Cela permet l'expression de gènes pro-inflammatoires et la sécrétion de cytokines (Figure 1) [5]. L'activation des TLR induit également celle de mTOR (mammalian target of rapamycin), conduisant ainsi à une augmentation de la synthèse protéique $[6,7]$. Il est admis depuis de nombreuses années que les acides aminés sont essentiels à l'activation de mTOR, vraisemblablement pour permettre à la cellule de coordonner l'abondance en acides aminés avec la voie de signalisation qui contrôle la synthèse protéique [8]. Dans les cellules inflammatoires, une augmentation de l'apport en acides aminés pourrait être essentielle pour assurer leur fonction.
V. Mieulet: Institut Curie, Inserm U830, bâtiment Trouillet-Rossignol,

26, rue d'Ulm, Paris, France.

R.F. Lamb : Cross cancer institute,

Department of oncology,

University of Alberta,

11560 University Avenue T6G 122 Edmonton,

Alberta, Canada.

virginie.mieulet@curie.fr

\section{Effets du régime alimentaire}

sur la réponse immunitaire induite par le LPS

Notre étude a décrypté un des mécanismes permettant de comprendre l'effet délétère d'une absence de nutriments sur la réponse immunitaire innée en identifiant les voies de signalisation régulées par un acide aminé en particulier. Nous avons tout d'abord analysé in vivo chez la souris l'effet de la mise à jeun sur la production de TNF $\alpha$ (tumor necrosis factor $\alpha$ ) induite par le LPS. La mise à jeun induit une diminution du taux plasmatique de TNF $\alpha$ corrélée à une diminution de l'activation de la voie MEK/ERK (mitogen activated protein kinase kinase/extracellular signal-regulated kinase) dans les cellules de Kuppfer (macrophages présents dans le foie constituant la réserve principale de macrophages de l'organisme) [9]. Ces résultats suggèrent que des variations de la concentration en

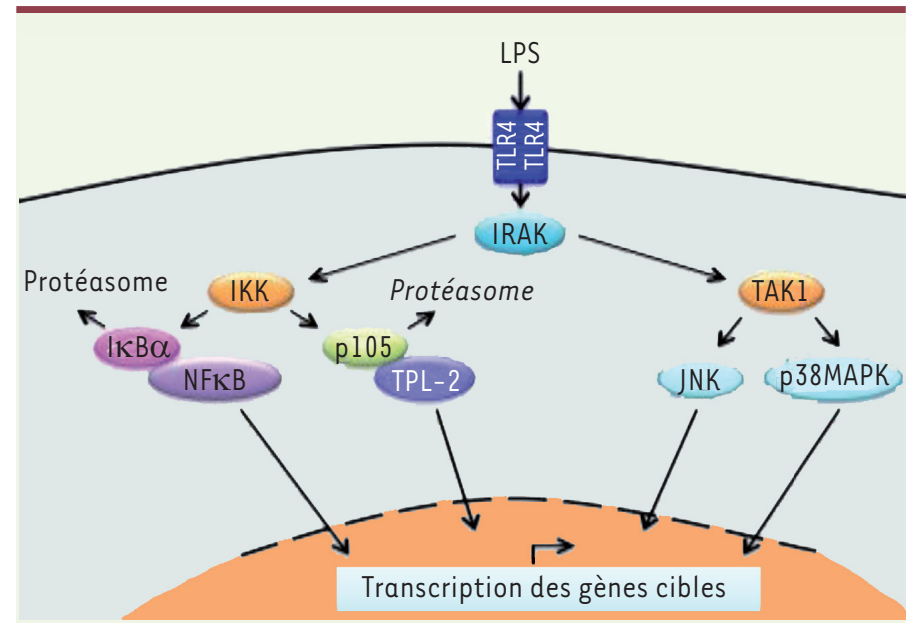

Figure 1. Activation de cascades de signalisation, en aval du TLR4, par le lipopolysaccharide (LPS) dans les macrophages. L'activation du Toll-like receptor 4 (TLR4) par le LPS dans les macrophages conduit à l'activation de la sérine thréonine kinase IRAK (Interleukin-1 [IL-1] receptor-associated kinase) en amont du complexe IKK (IKB kinase) et de la protéine kinase TAKI (TGF $\beta$-activated kinase). IKK phosphoryle les inhibiteurs IKB $\alpha$ et $\mathrm{p} 105$, ce qui induit leur dégradation par le protéasome et permet l'activation de NFKB et de la MAP3K TPL-2, respectivement. L'activation de TAKl conduit à l'activation des voies JNK et p38MAPK. L'activation de ces cascades de signalisation induite par l'activation du TLR4 par le LPS dans les macrophages permet la transcription de gènes impliqués dans l'immunité innée comme la sécrétion de cytokines. 


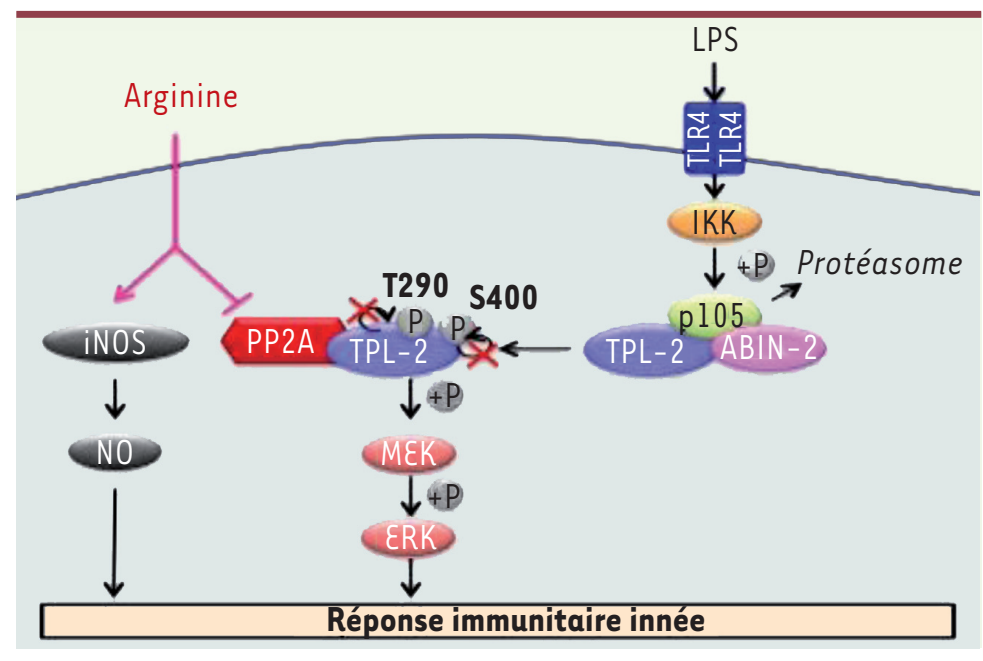

Figure 2. L'arginine contrôle MEK/ERK en réponse au LPS dans les macrophages via TPL-2. En l'absence de stimulus, la stabilité de TPL-2 est maintenue grâce à son interaction avec les protéines p105 et ABIN-2. Ce complexe ternaire inhibe l'activité kinase de TPL-2. L'activation du TLR4 par le LPS permet la libération de TPL-2 du complexe ternaire et sa phosphorylation sur plusieurs sites dont la Thr290 dans le domaine kinase et la Ser400 dans le domaine carboxyterminal de la protéine tous deux essentiels pour l'activité kinase de TPL-2. La présence d'arginine est nécessaire à l'activation de TPL-2 car elle prévient l'interaction de TPL-2 avec une phosphatase de type PP2A. En l'absence d'arginine, PP2A interagit fortement avec TPL-2 et déphosphoryle la protéine kinase sur les sites Thr290 et Ser400 conduisant ainsi à une inhibition de son activité et de la phosphoryla-

tion de son substrat MEK. L'arginine joue donc un rôle crucial dans la réponse immunitaire innée car en plus d'être le précurseur de monoxyde d'azote (NO), un puissant agent antimicrobien, elle contrôle MEK/ERK et la production de TNF $\alpha$ via la régulation de la kinase TPL-2.

nutriments durant la mise à jeun pourraient avoir un impact sur la voie de signalisation MEK/ERK dans les macrophages et donc sur la réponse inflammatoire induite par le LPS. Alors que le LPS active, via le TLR4, les cascades de signalisation MEK/ERK, NFKB, JNK et p38MAPK dans les macrophages en culture, les acides aminés ne sont requis que pour l'activation de MEK/ERK induite par le LPS [9]. Nous avons postulé que la sérine thréonine kinase TPL-2 (tumor progression locus 2), appelée aussi MAP3K8 (mitogen activated protein kinase kinase kinase 8), est essentielle à l'activation de MEK/ERK dans les macrophages. En effet, TPL-2 pouvait être une cible potentielle des acides aminés. La mesure de l'activité kinase de TPL-2 in vitro en réponse aux acides aminés a confirmé cette hypothèse [9]. La réponse aux acides aminés glutamine (acides aminés «branchés», leucine, valine et isoleucine) et arginine, connus pour activer la voie de mTOR [8], a été déterminée. Seule l'absence d'arginine prévient la phosphorylation de MEK induite par l'expression de TPL-2 dans les cellules 293T [9].

\section{Rôle de l'arginine sur l'activation} de TPL-2

Des études précédentes ont montré qu'en l'absence de stimulation, TPL-2 forme un complexe ternaire avec les protéines NFKBl pl05 et ABIN-2 (A-20 binding inhibitor of $N F K B-2)$ [10]. L'interaction de TPL-2 avec pl05 et $A B I N-2$ est nécessaire au maintien de sa stabilité [10]. En revanche, l'interaction de pl05 avec le domaine kinase de TPL-2 induit une inhibition de l'activité kinase de TPL-2, probablement en empêchant l'accès à son substrat MEK [11]. En réponse au LPS, TPL-2 est activée suite à une série d'événements dont la phosphorylation de pl05 par le complexe IKK (IKB kinase) et sa dégradation par le protéasome, ce qui permet la libération de TPL-2 du complexe ternaire et sa phosphorylation au niveau des sites Thr290 dans la boucle d'activation de son domaine kinase et Ser400 dans sa partie carboxy-terminale (Figure 2) $[12,13]$. La phosphorylation de ces deux sites est requise pour l'activité kinase de TPL-2 et résulterait d'une autophosphorylation [13, 14]. Nous avons montré que TPL-2 n'est pas phosphorylée en l'absence d'arginine [9]. Ceci serait dû à une augmentation de l'interaction de TPL-2 avec une protéine phosphatase de type PP2A observée en l'absence d'acides aminés [9]. L'arginine contrôle l'activation de MEK/ERK dans la réponse immunitaire innée via la régulation de la phosphorylation de TPL-2 par une phosphatase de type PP2A (Figure 2).

\section{L'arginine contrôle MEK/ERK} et la production de TNF $\alpha$ in vivo

Qu'en est-il du rôle de l'arginine dans la réponse immunitaire innée in vivo ? Bien que la mise à jeun chez la souris reflète les effets d'une absence en nutriments $a$ priori plus sévère que l'absence d'acides aminés orchestrée in vitro, seuls les taux de trois acides aminés que sont l'acide aspartique, l'alanine et l'arginine diminuent significativement chez les souris à jeun par rapport aux souris nourries. Cette variation est corrélée avec une diminution de l'activation de MEK/ERK dans les macrophages en réponse au LPS. De façon encore plus surprenante, seule l'administration orale d'arginine à des souris à jeun permet de restaurer l'activation de MEK/ERK dans les macrophages et la production de TNF $\alpha$ [9]. Dans les macrophages activés, la production d'oxyde nitrique (NO) à partir d'arginine par la forme inductible de NOS (inducible nitric oxyde synthase), est essentielle pour les mécanismes de défense de l'hôte contre de nombreux pathogènes [15]. Ainsi, outre la production de NO, un nouvel aspect critique du rôle de l'arginine dans la réponse immunitaire innée est dévoilé : 
l'arginine serait nécessaire à l'activation de MEK/ERK et à la production de cytokines (Figure 2).

L'arginine est un acide aminé semiessentiel car sa synthèse endogène est insuffisante pour permettre la croissance néonatale chez les mammifères et un apport exogène est nécessaire. L'arginine pourrait, en raison de sa synthèse limitée, également faire le lien entre statut nutritionnel et immunité innée. En l'absence de nutriments, l'arginine synthétisée par l'organisme serait suffisante pour assurer un niveau minimal d'activation de MEK/ERK dans les cellules inflammatoires. En revanche, un niveau supérieur d'activation de cette voie ne pourrait être atteint qu'avec un apport exogène d'arginine. Plus généralement, l'absence de nutriments peut avoir un effet délétère qui se traduit par exemple par l'augmentation de la susceptibilité aux infections. Inversement, la restriction calorique serait bénéfique au développement et au traitement de certains cancers [16, 17]. Un apport calorique excessif peut provoquer des maladies métaboliques. Par exemple, chez les sujets obèses, l'activation des macrophages infiltrés dans le tissu adipeux serait impliquée dans le développement d'une résistance à l'insuline corrélée à une augmentation de la concentration plasmatique de certains acides aminés dont l'arginine [18]. En activant la réponse immunitaire innée, l'arginine pourrait promouvoir le recrutement de macrophages au niveau du tissu adipeux et contribuer à une inflammation chronique caractéristique des sujets obèses. $\diamond$

Arginin and innate immune response

\section{CONFLIT D'INTÉRÊTS}

Les auteurs déclarent n'avoir aucun conflit d'intérêts concernant les données publiées dans cet article.

\section{RÉFÉRENCES}

1. Ambrus Sr JL, Ambrus Jr JL. Nutrition and infectious diseases in developing countries and problems of acquired immunodeficiency syndrome. Exp Biol Med $2004 ; 229$ : 464-72.

2. Moret $Y$, Schmid-Hempel P. Survival for immunity: the price of immune system activation for bumblebee workers. Science $2000 ; 290$ : 1166-8.

3. Chan J, Tian Y, Tanaka KE, et al. Effects of protein calorie malnutrition on tuberculosis in mice. Proc Nat Acad Sci USA 1996 ; 93 : 14857-61.

4. Delneste $Y$, Beauvillain C, Jeannin P. Immunité naturelle : structure et fonction des Toll-like receptors. Med Sci (Paris) 2007 ; $23: 67-74$.

5. West AP, Koblansky AA, Ghosh S. Recognition and signaling by toll-like receptors. Annu Rev Cell Dev Biol 2006 ; 22 : 409-37.

6. Lelouard H, Schmidt EK, Camosseto V, et al. Regulation of translation is required for dendritic cell function and survival during activation. J Cell Biol 2007 ; 179 : 1427-39.

7. Julien LA, Roux PP. mTOR, la cible fonctionnelle de la rapamycine. Med Sci (Paris) 2010 ; 26 : 1056-60.

8. Avruch J, Long X, Ortiz-Vega S, et al. Amino acid regulation of TOR complex 1 . Am J Physiol Endocrinol Metab 2009; 296 : ع592-602.
9. Mieulet V, Yan L, Choisy C, et al. TPL-2-mediated activation of MAPK downstream of TLR4 signaling is coupled to arginine availability. Sci Signal 2010 ; ra6l.

10. Lang V, Symons A, Watton SJ, et al. ABIN-2 forms a ternary complex with TPL-2 and NF-kappa B1 pl05 and is essential for TPL-2 protein stability. Mol Cell Biol $2004 ; 24$ : 5235-48.

11. Beinke S, Deka J, Lang V, et al. NF-kappaBl pl05 negatively regulates TPL-2 MEK kinase activity. Mol Cell Biol $2003 ; 23: 4739-52$.

12. Stafford MJ, Morrice NA, Peggie MW, Cohen P. Interleukin-1 stimulated activation of the COT catalytic subunit through the phosphorylation of Thr290 and Ser62. FEBS Lett $2006 ; 580: 4010-4$.

13. Robinson MJ, Beinke $S$, Kouroumalis $A$, et al. Phosphorylation of TPL-2 on serine 400 is essential for lipopolysaccharide activation of extracellular signalregulated kinase in macrophages. Mol Cell Biol 2007 ; $27: 7355-64$.

14. Handoyo H, Stafford MJ, McManus $\varepsilon$, et al. IRAK1independent pathways required for the interleukin1-stimulated activation of the Tpl2 catalytic subunit and its dissociation from ABIN2. Biochem J 2009; $424: 109-18$.

15. Nussler AK, Billiar TR, Liu ZZ, Morris SM Jr. Coinduction of nitric oxide synthase and argininosuccinate synthetase in a murine macrophage cell line. Implications for regulation of nitric oxide production. J Biol Chem 1994 ; 269 : 1257-61.

16. Kalaany NY, Sabatini DM. Tumours with PI3K activation are resistant to dietary restriction. Nature $2009 ; 458: 725-31$

17. Raffaghello L, Lee C, Safdie FM, et al. Starvationdependent differential stress resistance protects normal but not cancer cells against high-dose chemotherapy. Proc Natl Acad Sci USA 2008 ; 105 : 8215-20.

18. Newgard CB, An J, Bain JR, et al. A branchedchain amino acid-related metabolic signature that differentiates obese and lean humans and contributes to insulin resistance. Cell Metab 2009; 9 : 311-26.

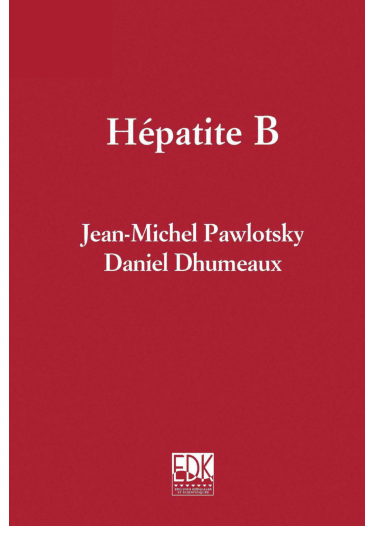

ISBN : 978-2-8425-4131-6 576 pages

\section{Bon de commande}

À retourner à EDK, 2, rue Troyon - 92316 Sèvres Cedex

Tél. : 0155641393 - Fax : 0155641394 - E-mail : edk@edk.fr

NOM :

Prénom :

Adresse :

Code postal :

Ville :

Pays :

Fonction :

Je souhaite recevoir l'ouvrage Hépatite B : $54 €+3 €$ de port $=\mathbf{5 7} €$ TTC offre exceptionnelle réservée aux abonnés à $\mathrm{m} / \mathrm{s}$ jusqu'au 31 décembre 2010

en ............... exemplaire, soit un total de …............................... €

$\square$ Par chèque, à l'ordre de $\mathbf{E} \mathbf{D} \mathbf{K}$

$\square$ Par carte bancaire : $\quad \square$ Visa $\square$ Eurocard/Mastercard

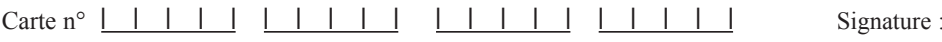

Date d'expiration: $\quad \underline{1 \mid 1} \underline{1|| \mid}$

$\mathrm{N}^{\circ}$ de contrôle au dos de la carte : 